\title{
Análisis del trabajo pedagógico realizado por docentes con estudiantes que presentan sordoceguera en cinco regiones de Chile, en escuelas regulares con Programas de Integración Escolar (PIE) ${ }^{1}$ y escuelas especiales
}

\author{
Donoso Araya, Oriana Elizabeth; Lattapiat Navarro, Pamela; Olivares Petit, Carla \\ Análisis del trabajo pedagógico realizado por docentes con estudiantes que presentan sordoceguera en cinco \\ regiones de Chile, en escuelas regulares con Programas de Integración Escolar (PIE) ${ }^{1}$ y escuelas especiales \\ Revista Educación, vol. 46, núm. 1, 2022 \\ Universidad de Costa Rica, Costa Rica \\ Disponible en: https://www.redalyc.org/articulo.oa?id=44068165014 \\ DOI: https://doi.org/10.15517/revedu.v46i1.45087
}

\section{(ㄷ) $(1) \Theta(9)$}

Esta obra está bajo una Licencia Creative Commons Atribución-NoComercial-SinDerivar 3.0 Internacional. 


\section{Análisis del trabajo pedagógico realizado por docentes con estudiantes que presentan sordoceguera en cinco regiones de Chile, en escuelas regulares con Programas de Integración Escolar (PIE) ${ }^{1}$ y escuelas especiales}

Analysis of Pedagogical Practices with Deaf-Blind Students in Five Regions of Chile at Both Integrated ${ }^{1}$ and Special Need Schools

Oriana Elizabeth Donoso Araya

Universidad Metropolitana de Ciencias de la Educación,

Chile

DOI: https://doi.org/10.15517/revedu.v46i1.45087 Redalyc: https://www.redalyc.org/articulo.oa?

oriana.donoso@umce.cl

(iD https://orcid.org/0000-0002-2269-9153

Pamela Lattapiat Navarro

Universidad Metropolitana de Ciencias de la Educación,

Chile

pamela.lattapiat@umce.cl

(iD https://orcid.org/0000-0003-0299-2288

Carla Olivares Petit

Universidad Metropolitana de Ciencias de la Educación,

Chile

carla.olivares@umce.cl

(iD) https://orcid.org/0000-0002-2366-4091

Recepción: 05 Enero 2021

Aprobación: 02 Marzo 2021

\section{Resumen:}

Este artículo se enmarca en un estudio sobre las competencias comunicativas de la población estudiantil sordociega y la interacción didáctica con sus docentes. Se finaliza con sugerencias didácticas en el ámbito de la sordoceguera y de la comunicación. El propósito es indagar el nivel de conocimiento que presenta cada participante del cuerpo docente, de escuelas especiales y de escuelas regulares con proyectos de inclusión, sobre esta discapacidad dual, de acuerdo con las interacciones didácticas que desarrollan con su estudiantado. La metodología es de tipo cualitativa, modalidad de estudio de casos a partir de una muestra de 12 personas, correspondientes a una población estudiantil sordociega en cinco regiones de Chile. Los hallazgos encontrados en el estudio de campo permitieron proponer sugerencias para la mejora de la comunicación y de las interacciones didácticas y, de este modo, aportar a los desafíos metodológicos que generan los aprendizajes de estas personas.

PALAbras ClaVE: Sordoceguera, Comunicación, Relación profesorado- estudiantado, Estrategias metodológicas.

\section{Abstract:}

This article encompasses one component of a study on communication skills among deaf-blind students and didactic interactions with their teachers. The objective of this field study was to examine the diferent proficiency levels of the teaching faculty with regards to this disability and based on didactic interactions with their students. The selected teachers either taught at special needs schools or integrated schools participating in the inclusion program. The study was based on a qualitative case study with a sample of twelve deaf-blind individuals from five regions of Chile. The conclusion provides specific teaching recommendations about deafblind communication and reveals the existence of knowledge gaps among teachers with regards to communicating with their deafblind students. Suggestions were made to improve communication and didactic interactions to overcome existing methodological teaching hurdles when teaching this population group.

KEYwORDS: Deaf-Blindness, Communication, Student-Teacher Relationship, Methodological Strategies. 


\section{INTRODUCCIÓN}

Diversos países de América Latina y el Caribe se encuentran trabajando con personas sordociegas. Si bien se ha avanzado significativamente en la educación de esta población, Romero et al. (2020), plantean: "que en la actualidad conviven la exclusión, la segregación, la integración y la inclusión” (p. 200).

La sordoceguera es una discapacidad dual, escasamente conocida en Chile, la cual es considerada como discapacidad única en Europa y Estados Unidos. En Latinoamérica solo Colombia y Perú han logrado este reconocimiento. Chile, a partir del año 2010, con la promulgación del Decreto $\mathrm{N}^{\circ} 170$, define esta discapacidad como permanente; en su artículo 73 expresa lo siguiente:

Se entenderá por multidéficit, en adelante discapacidades múltiples, la presencia de una combinación de necesidades físicas, médicas, educacionales y socio/emocionales y con frecuencia también, las pérdidas sensoriales, neurológicas, dificultad de movimientos y problemas conductuales que impactan de manera significativa en el desarrollo educativo, social y vocacional. Para los efectos de este reglamento la sordoceguera será considerada como discapacidad múltiple y constituye una discapacidad con características únicas, que se caracteriza por la existencia de una discapacidad auditiva y una discapacidad visual lo suficientemente severas como para afectar la comunicación, la movilidad y el acceso a la información y al entorno (Decreto $\mathrm{N}^{\circ} 170,2010$, art. 73, título IV).

De acuerdo con lo anterior, la población sordociega ha sido considerada como: personas con complicaciones para desarrollarse normalmente en la sociedad, por lo que muchas veces terminan siendo excluidas, pues tienen falencias para comunicarse y relacionarse con otras personas. Esto también se extrapola en el ámbito educativo, debido a que en esta área aún se observan problemas en la organización de clases. De igual forma, se hace relevante una definición de roles y responsabilidades de cada miembro que forme parte del equipo de trabajo, por ejemplo, tener un horario establecido para reunirse de manera cotidiana para proyectar las acciones del equipo, de forma conjunta. Estos aspectos, como describe Rodríguez (2014), han sido hallazgos que muestran grandes debilidades en la organización, estructura y gestión escolar en Chile.

Se observa, que el concepto de sordoceguera no se encuentra claramente definido, debido a que se considera como discapacidad múltiple, lo que agudiza la problemática en el trabajo del personal docente con el alumnado sordociego.

Este estudio de campo detecta que esta definición de sordoceguera no es totalmente comprendida por el cuerpo docente, dado que hay falta de conocimiento sobre esta discapacidad dual, así como de la ubicación del estudiantado en el sistema educacional, puesto que no existe un registro de personas con sordoceguera en Chile. El Servicio Nacional de la Discapacidad (SENADIS), organismo público, tiene por finalidad promover la igualdad de oportunidades, inclusión social, participación y accesibilidad de las personas con discapacidad; sin embargo, no ha hecho un catastro formal que considere esta condición (Araus, 2015).

Esta investigación abordará una dimensión teórica respecto a la sordoceguera, posteriormente, describe el método con que se organizó la investigación para profundizar en los resultados y, finalmente, mostrar la relevancia de la caracterización sobre la enseñanza y aprendizaje en un contexto inclusivo.

\section{MARCo TEÓRICO}

Para propiciar una mejor comprensión del estudio, se darán a conocer algunos elementos relevantes del concepto de sordoceguera y sus clasificaciones, para finalizar con el concepto de interacción didáctica.

González y Laborda (2015) realizan un estudio sistemático sobre el tema de la sordoceguera con énfasis en la comunicación. Aportan una visión general sobre 80 años de estudios de la sordoceguera, donde señalan las principales tendencias en investigación sobre este tema.

La Federación Española de Sordoceguera (2020) plantea que la sordoceguera es una discapacidad única, que tiene características y una serie de necesidades propias, derivadas de la pérdida de la capacidad visual y auditiva (Flores, 2021). 
La sordoceguera constituye una pérdida simultánea de visión y audición, afecta al conocimiento del entorno directo, por lo que limita la interacción del individuo y, por tanto, su desarrollo como persona en la sociedad (Sánchez y Benítez, 2012).

Del mismo modo, se puede encontrar que:

...una persona es sordociega cuando en ella se combinan dos deficiencias sensoriales (visual y auditiva) que se manifiestan en mayor o menor grado, generando problemas de comunicación únicos y necesidades especiales derivadas de la dificultad para percibir de manera global, conocer, y por tanto, interesarse y desenvolverse en su entorno. Como consecuencia de la incomunicación y desconexión con el mundo, que la privación sensorial genera en las personas sordociegas, éstas presentan una serie de dificultades que se manifestarán, de acuerdo a sus características; en la comunicación, en el acceso a la información, en la educación, en la capacitación profesional, en el trabajo, en la vida social y en las actividades culturales (Álvarez, 2004, p. 174).

Álvarez (s.f.) clasifica la sordoceguera en 4 tipos y la relaciona con la edad de adquisición: persona sordociega congénita, persona ciega congénita con sordera adquirida (persona ciegasorda), persona sorda congénita con ceguera adquirida (persona sordaciega) y persona sordociega tardía.

Es necesario destacar que de la pérdida visual y auditiva que presente la persona (sordociega total, sordociega con resto visual, sordociega con resto auditivo, sordociega con resto visual y auditivo; el origen congénito o adquirido; el orden y el modo en que perdieron los sentidos de la vista y del oído) dependerá el tipo de comunicación que se elija trabajar, y la forma en que este tipo de comunicación se use va a tener relación directa con el nivel de comunicación que la persona sordociega posea y de los apoyos que esta reciba durante el proceso educativo (Miles y Riggio,1999).

Además, se tiene que considerar que: "La sordoceguera, no es solo un problema de comunicación y percepción, sino que engloba todos estos factores y algunos más. La edad de adquisición de la sordoceguera es determinante del sistema de comunicación que utilice el sujeto" (Donoso, 2012, p. 66).

Para fundamentar el impacto de las pérdidas visuales y auditivas en el comportamiento de la persona, se hará referencia a las definiciones que otorga en Chile el Decreto No170 (2010), donde en su artículo 68 alude a la discapacidad de la visión como una alteración de la sensopercepción visual, la cual puede aparecer en diferentes grados y por diversas causas, lo cual implica consecuencias en la integración de una persona. Estas pérdidas visuales pueden ser de baja visión, donde se manifiesta una capacidad visual que es funcional para su vida con ayudas técnicas (remanente visual de 0.33 o menos en su medición central), y ceguera, donde su pérdida visual es tal que el remanente visual del mejor ojo con la mejor corrección (menor o igual a 0.05) no es funcional en su vida cotidiana, por lo cual debe hacer uso de sus otros sentidos para construir la realidad.

En relación con las pérdidas auditivas, el artículo 77 del mismo Decreto No170 (2010) considera limitaciones cuantitativas y cualitativas de la persona, que repercuten en su desarrollo y aprendizaje. Así, toma en cuenta el grado de pérdida auditiva como las dificultades que la persona pueda presentar, en su entorno inmediato y social. Según la pérdida auditiva, pueden existir personas con hipoacusia, es decir, con una pérdida parcial de la audición o con sordera, es decir, pérdida severa o total de la audición. En la evaluación se considera no sólo la perspectiva audiológica, sino que también la socioantropológica, en la cual las personas sordas conforman una comunidad particular con una lengua visogestual: la lengua de señas.

Estos elementos cualitativos y cuantitativos inciden en las formas de comunicación de estas personas. Se hace necesario destacar que en el grupo de estudio uno de los criterios de clasificación fue la edad de adquisición, elemento determinante del tipo de comunicación de esta población estudiantil, así como también, los apoyos familiares y profesionales que tuvieron.

La Deafblind International (DbI), en el documento Comprendiendo la sordoceguera, hace una clasificación de las personas sordociegas que se focaliza en su origen, de esta manera, es posible encontrar:

Personas sordociegas congénitas: nacen con problemas visuales y auditivos o adquirieron la sordoceguera antes del desarrollo del lenguaje.

Personas sordociegas adquiridas: pierden la audición luego de haber adquirido el lenguaje. 
Personas sordas o hipoacúsicas: pierden la visión o desarrollan un problema visual. En este último grupo se puede distinguir, entre aquellas personas sordas que utilizan la lengua de señas y desarrollan una pérdida visual y aquellas personas sordas que utilizan la lengua oral y desarrollan una pérdida de su visión. Como población sordociega adquirida también se encuentran aquellas personas que tuvieron audición y visión pero las pierden por algún accidente o enfermedad; asimismo, personas que son sordas o ciegas y desarrollan un impedimento del sentido que tenían indemne (Neri de Tronconis, 2009).

Álvarez (2004) comenta sobre lo compleja y heterogénea que es esta población, por la existencia de diversas variables y características individuales que comprenden cada una de las personas sordociegas, como el grado de pérdida, el momento de aparición, el nivel de comunicación, entre otras.

En la comunicación de la población infantil sordociega, el sentido del tacto constituye el sentido más importante. Con su cuerpo, la niñez adquiere experiencia de texturas, grosores, temperatura, peso, volumen, percepción del espacio, entre otros (Leonhardt et al., 1999). Sin embargo, se sabe que la consideración de los otros sentidos, así como los restos visuales y auditivos que la niña o niño posea, son imprescindibles para establecer la comunicación.

Se hace necesario profundizar en clasificaciones más específicas que orienten el quehacer docente; de tal manera, se seleccionó una clasificación funcional de las personas con sordoceguera que responde al momento y al orden de aparición de las deficiencias sensoriales y de la eficiencia de los restos funcionales de la persona, como lo plantea la definición de Sordociegos Internacional (DbI). Este grupo de personas defiende que la condición de sordoceguera sea determinada por la situación funcional, derivada de la dificultad comunicativa de la persona; dicha dificultad es resultado de la combinación de la deficiencia visual y auditiva. Utilizar solo el criterio cuantitativo no permite establecer un límite claro a partir del cual afirmar que una persona es sordociega, es decir, no hay determinados valores que equivalgan a ser persona sordociega, esto significa que es posible encontrar personas con los mismos valores de deficiencia pero con grados de autonomía distintos.

Definiciones de la sordoceguera según nivel de funcionamiento (Álvarez, 2004):

Bajo nivel de funcionamiento

Agrupa a niños, jóvenes y adultos de quienes se estima que su comunicación quedará limitada a aspectos básicos por no alcanzar lo que Fravell (1977) llama «motivación cognitiva». Describe el impulso/deseo infantil para interactuar con y aprender sobre el entorno por la simple búsqueda de conocimiento. Incluso cuando no existe la necesidad práctica de hacerlo y no hay refuerzo controlado externamente, ya sea social o material, para controlar esa conducta.

Nivel medio de funcionamiento

Agrupa a niños, jóvenes y adultos capaces de interesarse por el mundo cognitivamente (por las cosas y personas), capaces de generar estrategias para la resolución de problemas y de llevar una vida semi-independiente.

Alto nivel de funcionamiento

Agrupa a personas sordociegas sin otro límite cognitivo que el derivado de la propia sordoceguera y que demuestran estrategias de resolución de problemas e intereses que nos hacen pensar en ellos como susceptibles de llevar una vida y enseñanza normalizadas con las ayudas necesarias (p.114).

En el nivel de funcionamiento bajo, la intervención está orientada a desarrollar interacción con el entorno cercano de cada estudiante, lograr una comunicación estructurada y desarrollar autonomía personal. En el nivel de funcionamiento medio, el trabajo debe estar orientado a desarrollar un sistema de comunicación y habilidades sociales que le permita adquirir competencias para desenvolverse en un ambiente laboral protegido; en el nivel de funcionamiento alto, la labor educativa debe considerar siempre la introducción de contenidos académicos y culturales (Asociación de Sordociegos de España (ASOCIDE), s.f.).

Con la finalidad de profundizar el nivel de interacción en la comunicación del grupo en estudio se utilizaron como referencia los Niveles de Comunicación de Rowland y Stremel (1987), los cuales se muestran en la Tabla 1. 
TABLA 1

Modelo de secuencias de competencias de comunicación para personas sordociegas y multimpedidas sensoriales

\begin{tabular}{|c|c|}
\hline Niveles & Descripción \\
\hline $\begin{array}{l}\text { Nivel 1. Conducta pre } \\
\text { intencional Nivel } \\
\text { 2. Conducta } \\
\text { intencional Nivel } 3 . \\
\text { Comunicación pre- } \\
\text { simbólica no } \\
\text { convencional }\end{array}$ & $\begin{array}{l}\text { En el nivel 1, las manifestaciones que presenta cada } \\
\text { estudiante no indica una intención específica de } \\
\text { comunicación. La persona adulta que lo conoce interpreta sus } \\
\text { formas comunicativas. En el nivel 1, la conducta da a conocer } \\
\text { el estado del sujeto. Se observan movimientos de cabeza, } \\
\text { cambios posturales, movimientos de las extremidades y } \\
\text { expresión facial. La conducta sirve para influir en el } \\
\text { comportamiento de la persona que observa. Se perciben } \\
\text { movimientos corporales como: alcanzar un objeto, empujar, } \\
\text { de acercamiento y sonrisa. Uso de gestos no convencionales } \\
\text { con el fin de afectar a la persona que observa. Se notan los } \\
\text { movimientos gestuales del nivel } 2 \text {, además de manipular a } \\
\text { una persona y el contacto visual. }\end{array}$ \\
\hline $\begin{array}{l}\text { Nivel 4. Comunicación } \\
\text { Pre-simbólica } \\
\text { convencional } \\
\text { Nivel 5. Comunicación } \\
\text { simbólica concreta }\end{array}$ & $\begin{array}{l}\text { Evalúa el uso de formas de la comunicación a través de las } \\
\text { distintas señales y gestos convencionales e intencionales, los } \\
\text { cuales tienen intención de afectar las conductas de la otra } \\
\text { persona; movimiento corporal en otra persona (tocar, tomar la } \\
\text { mano, llevar hacia algún lugar), mirada y gestos con } \\
\text { intención, dirigirse a la localidad donde se desarrolla la } \\
\text { actividad, mostrar el objeto de la actividad preferida y gestos } \\
\text { naturales y simples (levantar los brazos para que lo tomen, } \\
\text { mirar el objeto que desea, asentir con la cabeza, agitar la } \\
\text { cabeza). Uso limitado de simbolos concretos con sentido } \\
\text { comunicacional para representar elementos del medio. Usos } \\
\text { de gestos representativos, por ejemplo: ven, siéntate. }\end{array}$ \\
\hline $\begin{array}{l}\text { Nivel 6. Comunicación } \\
\text { simbólica abstracta }\end{array}$ & $\begin{array}{l}\text { En la comunicación simbólica abstracta, los niños y niñas } \\
\text { usan símbolos abstractos o expresiones de una sola palabra } \\
\text { para comunicarse. El personal docente puede ampliar los } \\
\text { comentarios del estudiantado, existe un uso limitado de } \\
\text { simbolos concretos para representar objetos y entidades del } \\
\text { medio ambiente, correspondencia uno a uno entre símbolo y } \\
\text { la referencia. Esta comunicación se refiere a cuando la niña y } \\
\text { el niño usan el gesto natural, objeto o dibujo con sentido } \\
\text { comunicacional. }\end{array}$ \\
\hline $\begin{array}{l}\text { Nivel 7. Comunicación } \\
\text { simbólica formal }\end{array}$ & $\begin{array}{l}\text { Los niños y niñas comienzan a comprender las reglas } \\
\text { semánticas y de sintaxis de la lengua. Combinan dos o más } \\
\text { palabras para comunicarse, reorganizan las palabras para } \\
\text { cambiar el significado de una oración y hacen preguntas para } \\
\text { comunicar intenciones más sofisticadas. Combinación de dos } \\
\text { o más señas manuales. }\end{array}$ \\
\hline
\end{tabular}

Fuente: adaptación de Rowland y Stremel (1987).

Se requiere conocer los niveles comunicativos del estudiantado sordociego para establecer una interacción didáctica acorde a su nivel comunicativo.

La interacción didáctica se entenderá como "la comunicación intencional, estructurada y especializada para la enseñanza y el aprendizaje de conocimientos escolares" (Villalta et al., 2011, p.1143). Las personas autoras hacen referencia a seis niveles de la comunicación: 1. espacio - tiempo; 2. fases de la clase: inicio, desarrollo y cierre; 3 . secuencias temáticas; 4 . intercambios entre personas interlocutoras; 5 . intervenciones de cada interlocutor y interlocutora; 6. actos de habla.

Desde los postulados de Bronfenbrenner, la interacción didáctica debe tener en cuenta el ambiente ecológico en el cual la persona se encuentra inserta; esto comprende su espacio inmediato y todas las relaciones y roles con quienes integran su familia, amistades, docentes, entre otras personas que construyen, junto a la persona sordociega, su vida cotidiana (Torrico et al., 2002). 


\section{MÉTOdo}

El estudio se basa en un enfoque cualitativo, modalidad estudio de casos únicos, orientado bajo la referencia epistemológica del paradigma interpretativo, con una muestra de 12 personas sordociegas, pertenecientes a establecimientos educacionales regulares con Programa de Integración Escolar (PIE) de educación básica y secundaria. Además, escuelas especiales, caracterizadas por una acentuada cultura de trabajo con la población estudiantil con discapacidad, con la finalidad de describir los procesos de interacción educativa y las relaciones comunicativas entre profesorado y estudiantado. Los datos se recogieron a través de registro audiovisual, grupos focales, análisis de documentación y observaciones no participantes.

\section{Participantes}

A partir de los datos obtenidos, se definió una muestra de 12 personas sordociegas, estudiantes que presentaban uno de los cuatro tipos de sordoceguera nombradas anteriormente según Álvarez (2004).

La selección de la muestra se realizó sobre la base de una encuesta aplicada en las escuelas especiales y regulares con PIE, en 5 de las 16 regiones en que Chile divide su territorio para la organización interna del país. Esta selección tomó en cuenta la mayor densidad poblacional en esas regiones. Así, se seleccionaron: la Región Metropolitana, que constituye la capital del país; la Región de Valparaíso (V Región), la Región de O'Higgins (VI Región), la Región del Maule (VII Región) y la Región del Bío Bío (VIII Región). Los establecimientos educacionales fueron obtenidos de las bases de datos del Ministerio de Educación (MINEDUC) y del trabajo de campo de las investigadoras.

La encuesta aplicada fue una adaptación de un instrumento elaborado en México, por Guevara y Ramírez (2006), gracias al apoyo del Programa Hilton Perkins, a través de un subsidio de la fundación Conrad N. Hilton, instrumento denominado: Encuesta sobre Sordoceguera y Discapacidad Múltiple.

En la Tabla 2, se da cuenta de la individualización de las personas participantes de este estudio, su caracterización física (C1), tipos de sordoceguera (según etapa de adquisición de esta) y caracterización en niveles funcionales de desempeño (C2); lo anterior permite clasificar la muestra. De acuerdo con ASOCIDE (s.f.), estos criterios son útiles para estructurar la intervención pedagógica, dado que $\mathrm{C} 1$ lleva a comprender la modalidad de comunicación y C2 favorece la secuencia del programa según los aprendizajes esperados y conforme a las características del desempeño individual.

TABLA 2

Descripción del estudiantado

\begin{tabular}{lllll} 
Escuela & Sexo & Curso / nivel & $\begin{array}{l}\text { Caracterización física } \\
\text { [C1] }\end{array}$ & $\begin{array}{l}\text { Caracterización } \\
\text { funcional [C2] }\end{array}$ \\
\hline E1 & F & Plan laboral & Ciegasorda adquirida & Medio \\
\hline E1 & F & $1^{\circ}$ básico & Sordaciega congénita & Medio \\
\hline E1 & $\mathrm{M}$ & $7^{\circ}$ básico & Sordociego congénito & Medio \\
\hline E 2 & M & $2^{\circ}$ básico & $\begin{array}{l}\text { Sordo con ceguera } \\
\text { adquirida }\end{array}$ & Alto \\
\hline E3 & M & Nivel funcional & Sordociego congénito & Bajo \\
\hline E4 & M & Plan laboral & Sordociego congénita & Bajo \\
\hline E5 & M & $2^{\circ}$ básico & Sordociego congénito & Medio \\
\hline E6 & $\mathrm{M}$ & $8^{\circ}$ básico & Sordociego congénito & Alto \\
\hline ER7 & F & $8^{\circ}$ básico & Sordociega adquirida & Medio \\
\hline ER8 & M & $4^{\circ}$ medio & Sordociega adquirida & Alto \\
\hline CR9 & M & Centro de Salud & Sordociego adquirido & Alto \\
\hline & & & & Indeterminado No congénito \\
\end{tabular}


Fuente: Donoso et al., 2019.

La Tabla 2 muestra la cantidad de personas participantes sordociegas que cooperaron en esta investigación. Colaboraron mayoritariamente estudiantes de enseñanza básica, pero también se logró obtener participantes que pertenecían al plan laboral. En el Centro de Salud no fue posible observar ni registrar las actividades desarrolladas con el estudiante, por enfermedad de él mismo. En la primera columna se ubica la escuela especial, etiquetada como E, Escuela Regular como ER y Centro de Rehabilitación como CR.

Se puede observar que la Escuela (E1) tiene dos estudiantes sordociegas y uno sordociego, la Escuela (E6) tiene dos personas sordociegas y en las restantes escuelas solo hay una persona participante sordociega.

Esta investigación no solo abordó al estudiantado sordociego, sino también al cuerpo docente. A continuación, la Tabla 3 presenta los establecimientos y datos del personal docente que formaron parte de la investigación. En la primera columna aparece el tipo de escuela; en la segunda columna se visualiza el número de docentes participantes, así como asistentes de la educación. Finalmente, en la columna tres se encuentra la especialidad de cada profesional participante de la investigación y la formación que posee. 
TABLA 3

Distribución del profesorado y asistentes técnicas participantes por unidad educativa

\begin{tabular}{|c|c|c|}
\hline $\begin{array}{l}\text { Tipo de } \\
\text { escuela }\end{array}$ & $\begin{array}{l}N^{\circ} \text { de docentes y } \\
\text { asistentes } \\
\text { participantes }\end{array}$ & Profesión y formación en el área \\
\hline 1. E Especial & $\begin{array}{l}4 \text { educadoras } \\
\text { diferenciales } 1 \\
\text { profesora de básica }\end{array}$ & $\begin{array}{l}4 \text { educadoras diferenciales especialistas } \\
\text { en problemas de visión } 1 \text { profesora de } \\
\text { Ed. Básica en Educación Física } \\
\text { capacitada en Perkins }\end{array}$ \\
\hline 2. E Especial & 1 Educadora básica & $\begin{array}{l}1 \text { educadora de Educación Básica, } \\
\text { magister en Educación, sorda, con } \\
\text { competencia en lengua de señas chilena. }\end{array}$ \\
\hline 3. E Especial & $\begin{array}{l}1 \text { educadora } \\
\text { diferencial } 1 \\
\text { asistente }\end{array}$ & $\begin{array}{l}1 \text { educadora Diferencial, problemas de } \\
\text { visión /capacitada en Perkins } 1 \text { técnica } \\
\text { en Educación Diferencial }\end{array}$ \\
\hline 4. E Especial & $\begin{array}{l}1 \text { educador } \\
\text { diferencial } 1 \\
\text { asistente }\end{array}$ & $\begin{array}{l}1 \text { educador diferencial especialista en } \\
\text { déficit intelectual } 1 \text { técnica en Educación } \\
\text { Diferencial }\end{array}$ \\
\hline 5. E Especial & $\begin{array}{l}\text { 2 educadores } \\
\text { diferenciales } 1 \\
\text { asistente sorda }\end{array}$ & $\begin{array}{l}1 \text { educadora diferencial especialista } \\
\text { retardo mental /capacitada en Perkins } 1 \\
\text { candidato a magister Educación } \\
\text { Diferencial con mención en Necesidades } \\
\text { Múltiples Persona sorda usuaria de la } \\
\text { lengua de señas chilena }\end{array}$ \\
\hline 6. E Especial & $\begin{array}{l}\text { 2 educadoras } \\
\text { diferenciales } 1 \\
\text { asistente sorda }\end{array}$ & $\begin{array}{l}1 \text { educadora diferencial especialista } \\
\text { problemas de audición y lenguaje } 1 \\
\text { practicante profesional estudiante de Ed. } \\
\text { Diferencial especialista en problemas de } \\
\text { audición y lenguaje } 1 \text { técnica en } \\
\text { Educación Diferencial, persona sorda, } \\
\text { usuaria de la lengua de señas chilena }\end{array}$ \\
\hline 7. Escuela & 2 profesoras de & 2 docentes de las disciplinas Ciencias y \\
\hline Regular & educación media de & Lenguaje y Comunicación 1 \\
\hline PIE & $\begin{array}{l}\text { Ciencias y Lenguaje } \\
\text { y Comunicación } 1 \\
\text { educador } \\
\text { diferencial, } 1 \\
\text { intérprete en LSCh }\end{array}$ & $\begin{array}{l}\text { educador diferencial especialista en } \\
\text { problemas de audición y lenguaje } 1 \\
\text { educadora diferencial especialista en } \\
\text { problemas de audición y lenguaje/rol } \\
\text { intérprete. }\end{array}$ \\
\hline 8. Escuela & 2 educadores de & 2 educadores de Educación Básica, \\
\hline Regular PIE & $\begin{array}{l}\text { Básica Ciencias y } \\
\text { Lenguaje y } \\
\text { Comunicación } 1 \\
\text { asistente }\end{array}$ & $\begin{array}{l}\text { Ciencias y Lenguaje y Comunicación } 1 \\
\text { técnica en Educación Diferencial }\end{array}$ \\
\hline 9. Centro & $\begin{array}{l}\text { Profesionales de la } \\
\text { salud. }\end{array}$ & Jo $\mathrm{h}$ \\
\hline
\end{tabular}

Fuente: elaboración propia.

La tabla anterior muestra la cantidad de personal docente y asistente de la educación, participantes en el proceso de formación del estudiantado sordociego en esta investigación. El 52,1 \% son docentes de Educación Diferencial, el 17,3 \% son docentes de Educación Básica, el 8,6 \% son docentes de Educación Media, el 21,7 \% corresponde a Asistentes de la Educación, de quienes el $80 \%$ tiene Formación Técnica y el $20 \%$ no tiene formación profesional. Del total de asistentes, el $40 \%$ de personas son sordas usuarias de la lengua de señas chilena y se comunican con el estudiantado en LSCh táctil.

En cuanto a formación o capacitación sobre sordoceguera, del total de docentes que trabajan en las distintas escuelas, el 21,7 \% cuenta con esta formación. 


\section{Técnicas de recolección de datos}

Una vez identificada la muestra de personas sordociegas con la cual se trabajó, bajo los criterios éticos establecidos, se realizó: reuniones con los equipos de las escuelas que formaron parte del estudio de campo, firma de los consentimientos y asentimientos informados por parte de la población participante del estudio, personal directivo superior, docentes, padres, madres o personas tutoras; finalmente, se procedió a realizar el estudio de campo.

Las técnicas de recolección de datos fueron: observación de clases, registro audiovisual y desarrollo de grupos focales en las regiones VI de O'Higgins, VII del Maule, VIII del Bío-Bío y XIII Metropolitana de Santiago, a excepción de la $\mathrm{V}$ región de Valparaíso, en la cual no fue posible grabar las clases y efectuar grupos focales.

\section{Resultados}

La descripción de los resultados muestra una mirada sobre las rutinas de clases de la población estudiantil sordociega y cómo ella puede participar en el aula. Además, permite vislumbrar cuáles son las estrategias utilizadas por el personal docente en las asignaturas de Ciencias, y Lenguaje y Comunicación. Esta investigación es un primer acercamiento al contexto actual de salas de clases que atiende población estudiantil sordociega.

Para clarificar los resultados, a partir de los registros analizados, se establecieron dos categorías de análisis: la primera, remite a las competencias comunicativas y la segunda a la interacción didáctica.

Categoría 1. Competencias comunicativas en la clase de Lenguaje y Comunicación y en la clase de Ciencias, según las características físicas y funcionales de la población estudiantil sordociega.

El alumnado sordociego que forma parte del colectivo estudiado tiene dificultades en la comunicación, la socialización y el acceso a la información, así como carencia de habilidades comunicativas en algunas personas docentes y asistentes de la educación que interactúan con este grupo.

El estudio ha implicado un mayor tiempo en el proceso de establecer categorías de análisis, especialmente en lo referido a los informes por profesionales especialistas en los diagnósticos visuales y auditivos, debido a que en muchas escuelas o establecimientos educacionales no cuentan con información como los tipos de sordoceguera, edades de adquisición: congénitas o adquiridas, si han nacido con sordoceguera sin restos visuales ni auditivos, o si han nacido con sordera y luego, han sufrido la pérdida de la visión, si han nacido con ceguera y después perdieron la audición; si actualmente, presentan resto visual o auditivo y la funcionalidad que tienen esos remanentes. Además, la muestra es diversa y heterogénea.

Categoría 2. Interacción didáctica entre profesionales y estudiantes en la clase de Ciencias y en la de Lenguaje y Comunicación.

En las clases observadas se aprecia una carencia de profesionales que utilizan estrategias metodológicas orientadas a lo multisensorial. "El tacto, oído, el gusto y el olfato pueden actuar como canales de entrada a informaciones científicamente muy valiosas en la observación” (Soler, 1999, p.18). De acuerdo con Soler, es posible decir que por medios diversos se logran aprendizajes más significativos y enriquecedores; con la implementación de una didáctica multisensorial toda la población estudiantil se vería beneficiada, no solo las personas sordociegas de nacimiento.

En la interacción profesorado-estudiantado se observan dificultades en la mediación de los aprendizajes que se vinculan con la comunicación, educación basada en el movimiento y modelaje táctil. Lo anterior incide en las formas y funciones comunicativas del grupo participante, así como en los niveles de abstracción y simbolización en que se encuentran. 


\section{ANÁLISIS DE RESULTADOS}

\section{Competencias comunicativas:}

Con la finalidad de profundizar el nivel de interacción, en la comunicación del grupo en estudio, se utilizaron como referencia los Niveles de Comunicación de Rowland y Stremel (1987), lo cual permitió describir las potencialidades y desafíos de este grupo, ya que el nudo crítico es la interacción de la comunicación. Así, se describen sus etapas, según los siete niveles de competencia comunicativa que proponen estas personas autoras, para analizar la comunicación del grupo participante al interior de la sala de clases.

En el nivel 3: un estudiante se encuentra en dicha etapa, presenta sordoceguera congénita, asociada a parálisis cerebral. Ingresó tardíamente al sistema educacional especial y actualmente, a sus 14 años, es asistido permanentemente por una persona en el aula que interpreta sus formas y funciones comunicativas.

La auxiliar de este joven permanece en el aula y relaciona las actividades que propone la docente con los requerimientos del estudiante, cuyas formas de expresión son: gemidos, llantos, gritos, cambio de tono muscular, movimiento corporal, expresiones faciales. Sus manifestaciones se ubican entre los niveles 1. comportamiento pre-intencional y 3 . presimbólico no convencional.

En el nivel 4 se observó a una estudiante sordociega congénita, que presenta ceguera total y sordera profunda. Esta estudiante se integró a una escuela especializada en Chile a finales del año 2018, a la edad 12 años. Manifiesta muy descendidas sus habilidades comunicativas, dado que su proceso educativo ha sido muy irregular. Al inicio, su comunicación consistía en gritos y actitudes autoestimulatorias, gestos naturales que solo su madre interpretaba. Desde su ingreso al colegio a la fecha, se está trabajando con la metodología de calendarios (Blaha, 2001), con canasta de anticipación y estructuración de la rutina; hoy es capaz de permanecer sentada en actividades de alimentación y está reconociendo a las personas que trabajan con ella en la escuela.

En el nivel 6 se encuentran tres estudiantes, con uno se trabajó desde edades tempranas en escuela especializada, con estantería de anticipación y enfoque basado en el movimiento (Van Dijk, 1991), debido a que su escuela recibe apoyo de profesionales del programa Perkins International. Este estudiante, a sus 13 años, se comunica mediante clave objeto, gestos naturales y lengua de señas táctil. Desde el comienzo de su educación en la escuela, su madre ha desarrollado estrategias en el hogar guiada por el establecimiento educativo. Actualmente, tiene a una persona sorda usuaria de la Lengua de Señas Chilena al interior del aula, que comunica al estudiante lo que el profesorado realiza en clases.

Las otras dos personas estudiantes, una de sexo femenino y el otro masculino, que participaron en este estudio presentan formas primitivas de comunicación, tienen sordoceguera de origen congénita con restos visuales y auditivos, y portan el Síndrome de Charge. Este síndrome se encuentra entre una de las principales causas que generan sordoceguera congénita, como la prematuridad y la Embriopatía Rubeólica (Ruiz, 2017).

Se observa, en el caso del grupo estudiantil con Síndrome de Charge, que existen interacciones comunicativas con sus docentes; sin embargo, éstas son limitadas, especialmente, en los aspectos expresivos. Este grupo de estudiantes vocalizan y balbucean, no cuentan con un sistema alternativo y aumentativo de comunicación que sea más convencional y les permita desarrollar diálogos o conversaciones más complejas, a pesar de que sus niveles de comprensión indican que sí podrían hacerlo.

Se observa, al interior del aula, que el personal docente hace un esfuerzo por desarrollar el currículum nacional indicado por el Ministerio de Educación de Chile.

Para el nivel 7 se puede constatar que, en esta investigación, el grupo de estudiantes que se clasifica en esta etapa presenta uso de reglas estructuradas del sistema arbitrario de símbolos. Además de constatar las combinaciones ordenadas de acuerdo con las reglas del sistema de la lengua, tales como: palabras, sistema Braille, lengua de señas, dactilología en la mano y escritura. También se encuentra un estudiante preadolescente, con Síndrome de Usher tipo II, que nació sin presentar pérdidas sensoriales, pero estas se manifestaron hace dos años y su deterioro es significativo. Aún posee resto visual y auditivo, y se le está 
preparando para el implante coclear. Actualmente se le enseña el sistema Braille, que es un proceso lento en su adquisición y poco funcional, en su desempeño escolar.

En el presente estudio se observa que existe comunicación simbólica en el grupo seleccionado de estudiantes sordociegos, tanto en aquellos con sordoceguera tardía como en aquellos que nacieron con una discapacidad sensorial y en la adolescencia adquirieron la segunda discapacidad sensorial.

Cabe destacar que las habilidades comunicativas de este colectivo de estudiantes sordociegos, descienden paulatinamente en la medida en que sus canales sensoriales van perdiendo su funcionalidad.

Se hace indispensable aprovechar los remanentes visuales y auditivos que les permitan implementar otras formas de comunicación, dado que el conjunto de estudiantes de este estudio (Charge y Usher) presentan pérdidas progresivas.

Interacción didáctica:

Según Villalta et al. (2011), la interacción didáctica es entendida como aquella comunicación que se organiza, estructura y especializa al interior del currículum escolar. Estas personas autoras hacen referencia a seis niveles de la comunicación en sala de clase, nombrados previamente, de los cuales se tomó el que concierne a las fases de la clase: inicio, desarrollo y cierre. Junto con esto es indispensable conocer los recursos didácticos que utilice el personal docente, como calendarios, objetos, guías, material concreto, entre otros, que apoyen el desarrollo conceptual y los contenidos tratados.

Es relevante mencionar que el profesorado de las aulas observadas se encuentra alineado con el currículum nacional, enmarcado en las políticas educativas chilenas. Dada la heterogeneidad del estudiantado, se observa que la transmisión de los contenidos escolares no es recibida de igual forma por todas las personas, al no existir una diversificación curricular que dé respuesta a las necesidades particulares de cada estudiante que presenta sordoceguera, especialmente a lo referido al aprendizaje significativo y experiencial, adaptado a sus competencias comunicativas y niveles de funcionamiento.

Lo anteriormente señalado se comprende por la carencia de formación o capacitación sobre sordoceguera que presenta gran parte del personal profesional que formó parte del estudio. Del grupo, solo el 21,7 \% cuenta con una formación en el área.

En cuanto a las interacciones didácticas del equipo docente en el aula con sus estudiantes, y a partir de las observaciones del estudio de campo, estas fueron descritas tres etapas de la clase:

Inicio: el personal docente saluda a todo el grupo de la clase y en particular a la persona sordociega. Escasamente se observa que entre el estudiantado se saluden. En las actividades grupales, solo en dos establecimientos, el cuerpo docente saluda directamente al alumnado sordociego. En una actividad individual saluda al profesorado y a sus pares.

Presenta el contenido o temática a tratar en la clase; en las mayoría de las aulas el profesorado otorgó esta información de manera verbal; solo en tres escuelas, donde la población estudiantil sordociega tenía intérprete, docente o persona usuaria de lengua de señas tradicional o en su modalidad táctil, el mensaje se le entregaba a la persona sordociega.

La investigadora Miles (1995) recuerda que cuando se observa lo que una persona sordociega hace con sus manos, se puede entender mejor lo que podría saber sobre el mundo. El personal docente debe usar el método de mano sobre mano para guiar o modelar acciones, es decir, ofrecer objetos y ponerlos debajo de su mano, en lugar de tomar la mano de la otra persona y llevarla al objeto.

Esta sugerencia de Miles es fundamental para lograr una real comunicación con el estudiantado sordociego, pues el modelaje táctil entrega mucha información que debe ser complementada con las formas comunicativas, de cada persona sordociega.

La anticipación es la base para todo aprendizaje del estudiantado sordociego (Blaha, 2001), lo que implica que sea necesario contextualizar previamente cada clase o temática a tratar.

Desarrollo: en las aulas donde existe personal de apoyo para el estudiantado sordociego, el profesorado cumple un rol de mediador, por lo que resulta complejo para el profesorado adaptar algunos contenidos 
para que sean más cercanos a la experiencia de la población sordociega. Utiliza materiales para apoyar su comunicación, como calendarios, objetos concretos, entre otros. Asimismo, distribuye guías o materiales concretos diversos en conjunto con el personal asistente de apoyo, para interactuar de manera más individualizada con la población estudiantil sordociega.

En las aulas donde el trabajo es grupal y no hay asistente para el estudiantado sordociego, las indicaciones son generales, se observan dificultades en la interacción para que el estudiantado sordociego desarrolle sus aprendizajes; se advierten adaptaciones escasamente funcionales.

La participación de la persona sordociega en la sala de clases va a depender de la estimulación y de las oportunidades que el cuerpo docente y el personal asistente, le otorguen a cada individuo sordociego.

Sólo aquellas personas que son sordociegas tardías inician la comunicación de manera autónoma; el estudiantado que desde la infancia ha estado en escuelas especializadas y ha participado de un currículum funcional y ecológico, con una familia mediadora de sus aprendizajes, en conjunto con la escuela, logra también iniciar la comunicación de manera autónoma.

Se requiere un currículum funcional y un paradigma ecológico para responder a la diversidad, cambiar las conceptualizaciones tradicionales en el que toda la población menor hace lo mismo, en el mismo momento, de la misma forma y con los mismos materiales. Se requiere que las decisiones sean individuales, según las necesidades del estudiantado; estas han de ser funcionales y sobre la base de la realidad (Blanco, 1990).

Cierre: solo en una escuela se observaron conclusiones de la clase, las cuales mostraron resultados de la actividad desarrollada e hicieron preguntas al estudiantado sobre lo que hicieron o aprendieron y de lo que verán en la clase próxima. En todas las otras clases observadas, la actividad termina cuando finaliza la hora de la actividad, sin observarse ningún cierre explícito en el que se comente el contenido tratado.

El personal docente solo se despide del estudiantado sordociego diciéndole que salga a recreo o que se encontrarán la próxima clase.

El currículum funcional debe tener un resultado que sea comprensible para cada persona, con el fin de que se obtenga un producto que él o ella pueda reconocer como tal.

\section{Conclusiones}

Si bien hay diferencias en el quehacer del personal profesional de educación especial y aquellos de escuelas regulares de educación, entre las escuelas especiales también existen marcadas diferencias.

Describir e identificar las competencias comunicativas en la clase de Lenguaje y Comunicación y en la clase de Ciencias, con técnica de catastro y observación cualitativa de personal experto y con registro audiovisual, es una instancia positiva para conocer, de manera inicial, la participación e integración del conjunto de estudiantes con sordoceguera en la sala de clases. Así como describir la etapa o nivel de comunicación que presentan con su entorno y cómo esto impacta en su proceso de aprendizaje.

En lo referente a la comunicación, se observa, según los registros obtenidos, que los mayores problemas en la interacción entre profesionales y estudiantes, derivan del desconocimiento general de la población profesional, de las formas y funciones comunicativas y de los niveles de abstracción de sus estudiantes.

Mayoritariamente, el grupo estudiantil en estudio que se encuentra en escuelas especiales se ubica en las categorías: presimbólico convencional y simbólico emergente, en relación a sus niveles de comunicación y abstracción.

El grupo estudiantil con sordoceguera en escuela regular con PIE, tiene un nivel de comunicación que está en los niveles simbólico concreto y abstracto; sin embargo, sus habilidades comunicativas descienden paulatinamente en función de las pérdidas sensoriales que presentan de manera progresiva, derivadas de la sordoceguera adquirida.

Describir la interacción entre profesionales y estudiantes en la clase de las asignaturas seleccionadas, mediante técnica de observación directa, registro de campo, grupos focales y registro de videos, permite 
conocer las prácticas formativas al interior del aula y proporcionar orientaciones al personal docente para integrar pedagógicamente rutinas didácticas a las clases y que den inicio a la integración de este conjunto de estudiantes.

A partir de esta investigación se pudo dar cuenta de los factores clave en la interacción didáctica en las aulas del alumnado con sordoceguera y, de acuerdo con ello, se detectan tres nudos críticos: en primer lugar, la carencia de información del conglomerado de profesionales sobre la sordoceguera. Posteriormente, el desconocimiento de las estrategias didácticas requeridas con esta población y, para finalizar, la dificultad de la comunicación en la interacción del conjunto de profesionales que trabajan con esta población estudiantil. Solo dos escuelas, que han tenido formación en discapacidad múltiple y sordoceguera, desarrollan una interacción acorde a las necesidades de sus estudiantes, por lo que responden a sus formas y funciones comunicativas, realizan rutinas, aplican un currículum funcional y utilizan modelaje táctil en el marco de un paradigma ecológico.

Por otro lado, en el trabajo con la familia, se observaron escasas instancias de incorporación de los padres, madres y personas tutoras en el quehacer docente.

\section{SugERENCIAS DIDÁCTICAS}

El análisis a partir de este estudio de campo sugiere considerar las propuestas teóricas de Van Dijk (1991), la didáctica multisensorial de las ciencias de Miquel Albert Soler (1999) y el paradigma ecológico de Bronfenbrenner (1976), no solo para abordar las clases de Lenguaje y Comunicación, y Ciencias, sino para abordar todos los procesos de enseñanza-aprendizaje, de la población de estudiantes con sordoceguera.

Van Dijk (1991) plantea que el desarrollo cognitivo de las personas sordociegas, especialmente los casos en los que la sordoceguera es congénita, sigue una pauta de desarrollo totalmente distinta, como consecuencia de la privación de experiencias, por falta de personas mediadoras, que faciliten y refuercen sus aprendizajes. Describe en su Enfoque Basado en el Movimiento, la importancia de las experiencias motoras y propone actividades basadas en el movimiento, denominadas de resonancia.

Las actividades de resonancia comienzan con intentos de la persona adulta de entrar en el mundo de la niñez, de forma delicada a través del movimiento, para que su evolución pueda integrarse y no ser solo fragmentos de información, por lo que se precisa la intervención del medio (las personas que interactúan a su alrededor) para poderla integrar de manera completa.

Por otro lado, Sánchez y Benítez (2012) plantean la necesidad de utilizar todos los canales sensoriales, para que la población estudiantil acceda a la información y pueda desarrollar aprendizajes significativos.

El alumnado sordociego aprende en ambientes naturales, de allí la importancia de aplicar el paradigma ecológico de Bronfenbrenner, donde "los ambientes naturales son la principal fuente de influencia sobre la conducta humana” (Torrico et al., 2002, p.46). El estudiantado sordociego desarrolla su potencial en ambientes conocidos y naturales, por lo que las actividades deben ser funcionales y útiles para su vida.

Por otro lado, Bárbara Miles (1995) plantea que la intervención didáctica sea persona a persona, se utilice el modelaje táctil y que el diseño curricular sea funcional.

Es por ello que se recomienda el trabajo en equipo transdisciplinario; de tal manera que todo el equipo profesional que trabaje con el estudiantado sordociego, pueda compartir sus conocimientos y estrategias de intervención en pos del desarrollo del alumnado, en consideración de un horario establecido para reunirse de manera cotidiana (Rodríguez, 2014). Este equipo docente transdisciplinario debe incorporar rutinas que permitan facilitar el desarrollo del lenguaje y la comunicación, otorgar oportunidades al estudiantado de practicar sus interacciones, hacer preguntas abiertas, ampliar las expresiones del alumnado en sus diversas formas comunicativas, utilizar estructuras más complejas, aumentar su vocabulario y que este sea siempre significativo de su ambiente cotidiano. 
Para finalizar, como resultado de los hallazgos de esta investigación se considera necesario capacitar a docentes de escuelas especiales y escuelas regulares con PIE, en la temática de la sordoceguera. Asimismo, se considera relevante que los establecimientos de educación superior, que forman profesionales de la educación, den preparación en la temática de la sordoceguera. Por otra parte, se considera necesario fortalecer el trabajo colaborativo en las escuelas, el desarrollo de proyectos transdisciplinarios y la resolución conjunta de problemas. En este sentido, se hace indispensable formar redes de apoyo, no sólo al interior de los colegios, sino entre los distintos grupos de profesionales que trabajan con estudiantes que presentan sordoceguera: padres, madres, personas tutoras y el personal de investigación en el área; de tal manera, se podría crear nuevo conocimiento que sea base para mejoras en la educación y en la calidad de vida de la población sordociega y de sus familias.

\section{REFERENCIAS BIBLIOGRÁFICAS}

Álvarez, D. (2004). La sordoceguera: Una discapacidad singular. En P. Gómez y E. Romero (Coords.), La sordoceguera: Un análisis multidisciplinar (pp. 135-191). Organización Nacional de Ciegos Españoles (ONCE). https://dial net.unirioja.es/servlet/libro?codigo $=6299$

Álvarez, D. (s.f.). La comunicación expresiva y receptiva en las personas sordociegas. https://tinyurl.com/p6j8wna

Araus, M. (2015, 25 de junio). Sordociegos en Chile: La realidad que nadie ve ni escucha. El Definido. https://tinyu rl.com $/ 3 \times 863678$

Asociación de Sordociegos de España [ASOCIDE]. (s.f.). Las personas sordociegas. https://tinyurl.com/499fkpuj

Blanco, R. (1990). La atención a la diversidad en el aula y adaptaciones del currículo. En Palacios, G., Marchesi, A. y Coll, C. (Eds.), Desarrollo psicológico y educación. (pp. 411-438). http://www.juntosconstruyendofuturo.org

Blaha, R. (2001). Calendarios. Para estudiantes con múltiples discapacidades incluido Sordoceguera. Texas School for the Blind and Visually Impaired.

Bronfenbrenner, U. (1976). The Experimental Ecology of Education. Educational Researcher, 5(9), 5-15. https://do i.org/10.3102/0013189X005009005

Decreto $\mathrm{N}^{\circ} 170$. (2010). Fija normas para determinar los alumnos con necesidades educativas especiales que serán beneficiarios de las subvenciones para educación especial. Ministerio de Educación. República de Chile. En Biblioteca del Congreso Nacional, [en línea]. http://bcn.cl/1uvr8

Donoso, O. (2012). Sordoceguera y Necesidades Educativas Múltiples en Chile: Discapacidad Múltiple. Editorial Académica Española.

Donoso, O., Lattapiat, P. y Olivares, C. (2019). Exploración de la enseñanza para el aprendizaje en el contexto inclusivo: Los sordociegos en la clase de Ciencias y de Lenguaje y Comunicación. Reporte de Investigación Proyecto PMIEXA/PNII/14/2017. MINEDUC-UMCE (no publicado).

Flores, C. (2021). La sordoceguera en la educación actual: la educación física como apoyo a la inclusión. Revista Internacional de Apoyo a la Inclusión, Logopedia, Sociedad y Multiculturalidad, 7(1), 129-135. https://dx.doi.o rg/10.17561/riai.v7.n1.10

Fravell, J. (1977). Cognitive development. Englewood Cliffs, N.J.: Pentice-Hall. En Álvarez, D. (2004). La sordoceguera: Una discapacidad singular. En P. Gómez y E. Romero (Coords.), La sordoceguera: Un análisis multidisciplinar (pp. 135-191). Organización Nacional de Ciegos Españoles (ONCE). https:// dialnet.unirioja.es/servlet/libro?codigo $=6299$

González, H. y Laborda, C. (2015). Sordoceguera: estado de la cuestión en investigación socioeducativa sobre comunicación. Revista nacional e internacional de educación inclusiva, 8(2), 90-106. https://tinyurl.com/bds53 $\mathrm{m} 96$

Guevara, I. y Ramírez, M. (2006). Encuesta sobre Sordoceguera y Discapacidad Múltiple. Programa Hilton PerkinsFundación Conrad N. Hilton (no publicado) 
Oriana Elizabeth Donoso Araya, et al. Análisis del trabajo pedagógico realizado por docentes con e...

Leonhardt, M., Cantavella, F. y Tarragó, R. (1999). Iniciación del lenguaje en niños ciegos. Un enfoque preventivo. Organización Nacional de Ciegos Españoles (ONCE).

Miles, B. (1995). Perspectiva general sobre la sordoceguera. Centro de Información Nacional sobre Niños que son Sordos y Ciegos. http://documents.nationaldb.org/products/over-span.pdf

Miles, B. y Riggio, M. (Eds.). (1999). Conversaciones extraordinarias. Una guia para desarrollar una comunicación significativa con los niños y jóvenes con sordoceguera. https://docer.com.ar/doc/1s0s8c.

Ministerio de Educación. (s.f.). Incorporación de estudiantes PIE. https://tinyurl.com/22st73hc

Neri de Tronconis, M. (2009). Guia de Apoyo para la Sordoceguera. Fundación Empresas Polar, Socieven, Sordociegos de Venezuela.

Rodríguez, F. (2014). La co-enseñanza, una estrategia para el mejoramiento educativo y la inclusión. Revista Latinoamericana de Educación Inclusiva, 8(2), 219-233. https://dialnet.unirioja.es/servlet/articulo?codigo=49 94333

Romero, S., Ferioli, G., Fava, A., Nassif, M.E. y Cipollone, M. (2020). Prácticas educativas facilitadoras de aprendizajes de niños con discapacidad múltiple y sordoceguera. Anuario digital de investigación educativa, (3). http://revist as.bibdigital.uccor.edu.ar/index.php/adiv/article/view/4206

Rowland, C. y Stremel, K. (1987). Share and share alike: Conventional gestures to emergent language for learners with sensory impairments. [Compartir y compartir por igual: Gestos convencionales al lenguaje emergente para los estudiantes con discapacidades sensoriales] En: L. Goetz, D. Guess y K. Stremel (Eds.), Innovative program design for individuals with dual sensory impairments (pp. 56-57). Paul H. Brookes. https://tinyurl.com/e3nu5muf

Ruiz, M. (2017). La sordoceguera, su tratamiento normativo y atención a las personas que la presentan. Siglo Cero. Revista Española sobre Discapacidad Intelectual, 47(3), 29-54. https://doi.org/10.14201/scero20164732945

Sánchez, J.I. y Benítez, J.M. (2012). Sordoceguera: posibilidades de investigación psicoeducativa. International Journal of Developmental and Educational Psychology, 3(1), 249-259. http://www.redalyc.org/articulo.oa?id=3498323 38025

Soler, M. A. (1999). Didáctica multisensorial de las ciencias: Un nuevo método para alumnos ciegos, deficientes visuales y también sin problemas de visión ( $2^{\circ}$ Edición). Ediciones Paidós Ibérica.

Torrico, E., Santín, C., Andrés, M., Menéndez, S. y López, M. (2002). El modelo ecológico de Bronfrenbrenner como marco teórico de la Psicooncología. Anales depsicología, 18(1), 45-59. https://tinyurl.com/mtxt9y94

Van Dijk, J. (1991). Persons handicapped by rubella. Victors and victims. A follow-up study. [Personas discapacitadas por la rubéola. Víctimas y damnificados. Un estudio de seguimiento]. Swets \& Zeitlinger.

Villalta, M. A., Martinic, S. y Guzmán, M. A. (2011). Elementos de la interacción didáctica en la sala de clase que contribuyen al aprendizaje en contexto social vulnerable. Revista mexicana de investigación educativa, 16(51), 1137-1158. https://tinyurl.com/357jr2hp

\section{Notas}

[1] El PIE se trata de una estrategia educativa que proporciona una serie de recursos humanos y materiales de apoyo para las instituciones escolares, según las necesidades que pueda tener el estudiantado, en su trayectoria educativa (Ministerio de Educación de Chile. (s.f.)).

\section{INFORMACIÓN ADICIONAL}

Cómo citar: Donoso-Araya, O. E., Lattapiat-Navarro, P. y Olivares-Petit, C. (2022). Análisis del trabajo pedagógico realizado por docentes con estudiantes que presentan sordoceguera en cinco regiones de Chile, en escuelas regulares con Programas de Integración Escolar (PIE) y escuelas especiales. Revista Educación, 46(1). http://doi.org/10.15517/revedu.v46i1.45087 